\title{
Reversible Addition-Fragmentation Chain Transfer (RAFT) Bulk Polymerization of Styrene : Effect of R-Group Structures of Carboxyl Acid Group Functionalized RAFT Agents
}

\author{
Jung Min Lee, Ok Hyung Kim, Sang Eun Shim, Byung H. Lee, and Soonja Choe* \\ Department of Chemical Engineering, Inha University, Inchon 402-751, Korea
}

Received February 14, 2005; Revised April 11, 2005

\begin{abstract}
Three dithioester-derived carboxyl acid functionalized RAFT(reversible addition-fragmentation chain transfer) agents, viz. acetic acid dithiobenzoate, butanoic acid dithiobenzoate and 4-toluic acid dithiobenzoate, were used in the RAFT bulk polymerization of styrene, in order to study the effects of the R-group structure on the living nature of the polymerization. By conducting the polymerization with various concentrations of the RAFT agents and at different temperatures, it was found that the R-group structure of the RAFT agents plays an important role in the RAFT polymerization; the bulky structure and radical stabilizing property of the R-group enhances the living nature of the polymerization and allows the polymerization characteristics to be well controlled.
\end{abstract}

Keywords: RAFT agent, bulk polymerization, styrene, living radical polymerization.

\section{Introduction}

As the applications of polymeric materials have been expanded to information, microelectronics, and bio/medical sciences etc., the precise molecular design of polymers has become more significant. Among various polymerization methods developed to synthesize well-controlled molecular structure, the living free-radical polymerizations (LFRPs) have been received an extensive attention due to their effectiveness and simple process, thus several mechanisms have been proposed.

The living free-radical polymerization (LFRP), triggered by a pioneering research of Otsu ${ }^{1}$ is quite efficient in preparing controlled molecular architectures of various polymers. The LFRPs, which simulate ionic genuine living polymerizations, have been developed to synthesize well-controlled polymers having a PDI value close to unity. The LFRPs possess advantages over ionic polymerizations in the respect of versatile use of various monomers and their facile process. With the advent of several revolutionizing LFRPs, the synthesis of well-defined polymers/copolymers having desired structures has become realized in a convenient way. ${ }^{2}$

Based on the radical capping mechanism and agents, LFRPs are generally classified into nitroxide-mediated polymerization (NMP), ${ }^{3,4}$ atom transfer radical polymerization (ATRP), ${ }^{5-7}$ and reversible addition-fragmentation chain

*e-mail: schoe@inha.ac.kr

1598-5032/06/236-07@ 2005 Polymer Society of Korea transfer $(\mathrm{RAFT})^{8-10}$ polymerization. Among several LFRPs, RAFT technique utilizes a chain transfer agent (CTA) in order to control the molecular evolution. The most frequently used CTAs are dithioester-derivatives, $\mathrm{S}=\mathrm{C}(\mathrm{Z})$-SR. This type of CTA reacts with either a primary radical produced by an initiator or a propagating polymer species. In this process, controlled polymerization is achieved by the alternation of the activation and deactivation of the CTA between dormant and active moieties as shown in Scheme I.

Basic structure of RAFT agent is the following.

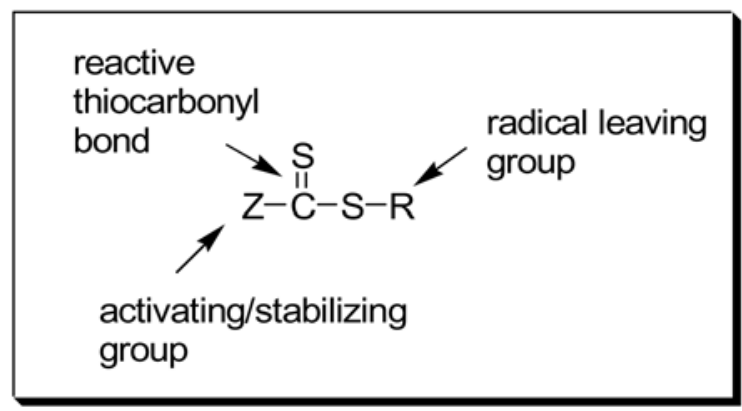

Here, $\mathrm{Z}$ is an active/stabilizing group, $\mathrm{C}=\mathrm{S}$ bond is a reactive thiocarbonyl bond, and $\mathrm{R}$ is a radical leaving group. As the polymerization proceeds, reversible addition- fragmentation step occurs on the two sulfur atoms. Both $\mathrm{R}$ and $\mathrm{Z}$ groups in the RAFT agent enhance the efficiency of reversible addition-fragmentation process. Especially, R-group should have a good leaving and facile re-initiation ability in 


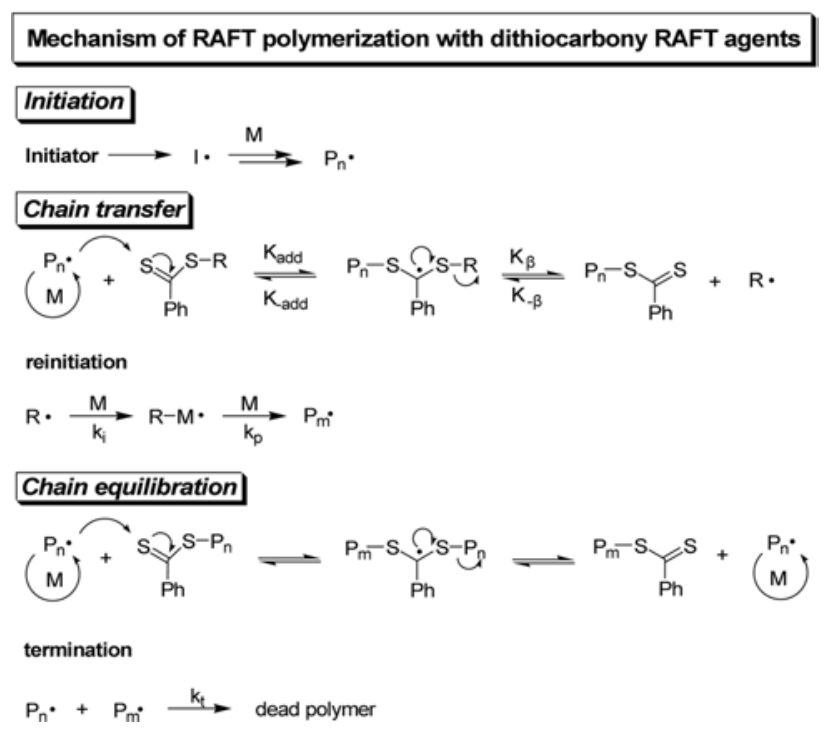

Scheme I. General mechanism of RAFT polymerization.

order to serve as a better RAFT agent. ${ }^{11-15}$ Z-group should have capability to activate and stabilize intermediate radicals, R·, generated during the polymerization. ${ }^{16,17}$ Furthermore, the end-functionalized macromolecules could be achieved by attaching various functional moieties to Rgroup. For example, water-soluble polymers have been synthesized using RAFT agents having carboxyl acid in Rgroup. ${ }^{18,19}$ In addition, we have reported that the carboxyl group functionalized RAFT agent improves the stability of polymer colloids prepared by miniemulsion polymerization. ${ }^{20}$

In this article, three different RAFT agents having same Z-group and carboxyl acid derived functional group in the R-group were employed in bulk polymerization of styrene and the effect of the structure of R-group was investigated based on the living nature, molecular weight evolution and molecular weight distribution (PDI; polydispersity index).

\section{Experimental}

Materials. Styrene (Junsei Chemical, Japan) was purified using an inhibitor removal silica column (Aldrich) and stored at $-5^{\circ} \mathrm{C}$ prior to use. 2,2-Azobis(isobutyronitrile) (AIBN; Junsei) was used without purification. Tetrahydrofurane (THF) used in synthesis was freshly distilled using sodium and benzophenone. Phenylmagnesium bromide, carbon disulfide, $\gamma$-butyrolactone, and $\alpha$-bromo- $p$-toluic acid were purchased from Aldrich Co. Silica used in column chromatography was Kieselgel-60 (Merck, Germany).

Synthesis of the RAFT Agents. Butanoic acid dithiobenzoate (CTA2) was synthesized by modifying the procedures described elsewhere. ${ }^{21}$ Phenylmagnesium bromide $(5 \mathrm{~g})$ is reacted with a solution of carbon disulfide $(1.98 \mathrm{~mL})$ in dry THF $(60 \mathrm{~mL})$ under the nitrogen atmosphere at $0{ }^{\circ} \mathrm{C}$. The colorless Grignard complex immediately turns yellowish red. After the addition of carbon disulfide is completed, the temperature of the mixture is allowed to rise gradually to room temperature. After stirring at room temperature for $0.5 \mathrm{~h}$, the resultant is refluxed for $1 \mathrm{~h}$ and cooled to room temperature. A solution of $\gamma$-butyrolactone $(20.18 \mathrm{~mL})$ in THF $(20 \mathrm{~mL})$ is then added dropwise over a period of $0.5 \mathrm{~h}$. After standing for $0.5 \mathrm{~h}$ at room temperature, the resulting mixture is heated under stirring at reflux temperature of the solvent, THF. Later, the reaction mixture is cooled with ice and terminated with water and ice-cold hydrochloric acid $(1: 1 \mathrm{v} / \mathrm{v}, 100 \mathrm{~mL})$, then the organic layer is separated employing ether. The aqueous layer is repeatedly extracted with ether $(25 \mathrm{~mL})$ and the combined organic layer is repeatedly extracted with ice-cold $10 \%$ aqueous sodium hydroxide $(30 \mathrm{~mL})$. The alkaline extracts upon acidification under ice-cold conditions furnish the crude dithioester as a foul smelling red oil, which is purified by column chromatography (silica 60 230 mesh, 20\% ethylacetate $/ n$-hexane) to give butanoic acid dithiobenzoate $(2.78 \mathrm{~g}$, yield: $42 \%)$ as a red solid. ${ }^{1} \mathrm{H}-\mathrm{nmr}\left(\mathrm{CDCl}_{3}\right)$ : 2.0-2.2 (m, $\left.2 \mathrm{H}, \beta-\mathrm{CH}_{2}\right), 2.4-$ 2.6 (t, $\left.2 \mathrm{H}, \gamma-\mathrm{CH}_{2}\right), 3.4-3.6\left(\mathrm{t}, 2 \mathrm{H}, \alpha-\mathrm{CH}_{2}\right), 7.3-7.7$ and 7.98.1 (m, $5 H$, phenyl).

Synthesis of 4-toluic acid dithiobenzoate (CTA3) was followed our previous publication. ${ }^{20}$

Polymerization of Styrene. A solution containing styrene (40 mL, $0.35 \mathrm{~mol})$, AIBN (0.0112 g, $0.068 \mathrm{mmol})$, and CTA was prepared under anhydrous condition. The solution was degassed by nitrogen for $15 \mathrm{~min}$. Aliquots $(5 \mathrm{~mL})$ of the mixture were transferred to scintillation vials. Then, the vials were sealed and completely sank in oil bath at the desired temperature for specified duration. After the polymerization, the reaction mixture was dissolved in THF and the polymer was precipitated in methanol and purified by repeated precipitations. Product was dried in vacuum oven.

Characterizations. The fractional conversion was calculated gravimetrically. The chemical structure of the synthesized RAFT agents was confirmed by Varian $400-\mathrm{MHz}{ }^{1} \mathrm{H}-$ NMR using $\mathrm{CDCl}_{3}$ as the solvent. The molecular weight and polydispersity index (PDI) were characterized using Waters GPC (Gel Permeation Chromatography) equipped with 510 differential refractometer and Viscotek T50 differential viscometer. $10^{5}, 10^{3}$, and $10^{2} \AA \mu$-styragel packed high-resolution columns were employed. Universal calibration curve was obtained using ten polystyrene standard samples (Polymer Laboratories, UK) with molecular weight ranging 580$7,500,000 \mathrm{~g} / \mathrm{mole}$. Polystyrene dissolved in THF was injected at a flow rate of $1.0 \mathrm{~mL} / \mathrm{min}$ and the elution volume was measured for calculation of the molecular weight.

\section{Results and Discussion}

Synthesis of RAFT Agents and the Selection. As discussed earlier, each moiety of RAFT agent plays a different role in polymerization involving addition-fragmentation 
process. Therefore, the proper molecular design of RAFT agent is critical for successful RAFT polymerizations..$^{20,22}$

The CTA1, purchased from Aldrich, contains one carbon atom next to the carboxyl acid functionality in R-group. The CTA2 contains three aliphatic carbons and the CTA3 carries a benzyl group in R-group, respectively. ${ }^{20,22}$

All of these three CTAs were started from Grignard reagent and the chemical name for the CTA1, CTA2, and CTA3 are acetic acid dithiobenzoate, butanoic acid dithiobenzoate, and 4-toluic acid dithiobenzoate, respectively. The characteristic singlet peak of R-group for CTA1 is observed at $4.2 \mathrm{ppm}$ for 2 protons on $\alpha$-carbon. In the case of CTA2, the ring opening of $\gamma$-butyrolactone is carried out and the CTA2 and CTA3 turned out to be red solid powder after purification. It is noted that the hydrogen atoms on carboxyl group expected to be observed above $10 \mathrm{ppm}$ was not discernable due to quite weak signal intensity. ${ }^{23}$

The Effect of the RAFT Agent Structures. Table I lists the number-average molecular weight $\left(M_{n}\right)$ and the polydispersity index (PDI) of the polystyrene prepared by RAFT bulk polymerization using the various RAFT agents with the 5.0 molar ratio of [RAFT]/[AIBN] at 60,70 , and $80^{\circ} \mathrm{C}$. The polymerization time is fixed for $96 \mathrm{hrs}$ to have substantially high conversion of around $80 \%$.

Figures 1, 2 and 3 represent the $M_{n}$ and PDI evolution with respect to the fractional conversion of the polystyrene prepared by RAFT bulk polymerization with [RAFT]/ $[\mathrm{AIBN}]=5.0$ using various RAFT agents, CTA1, CTA2, and $\mathrm{CTA} 3$, at 60,70 , and $80^{\circ} \mathrm{C}$, respectively. In three figures, controlled features of the polymerizations are dependant on various CTAs. The CTA2 exhibits the worst ability to control the polymerization, by showing the non-linearity in molecular weight-conversion relationship and PDI between 2.09 and 2.45. On the other hand, the CTA1 is better than CTA2 and CTA 3 is believed to be fairly good RAFT agent due to showing linearity between the $M_{n}$ and conversion, and varying the PDI between 1.36 and 1.40 , and between

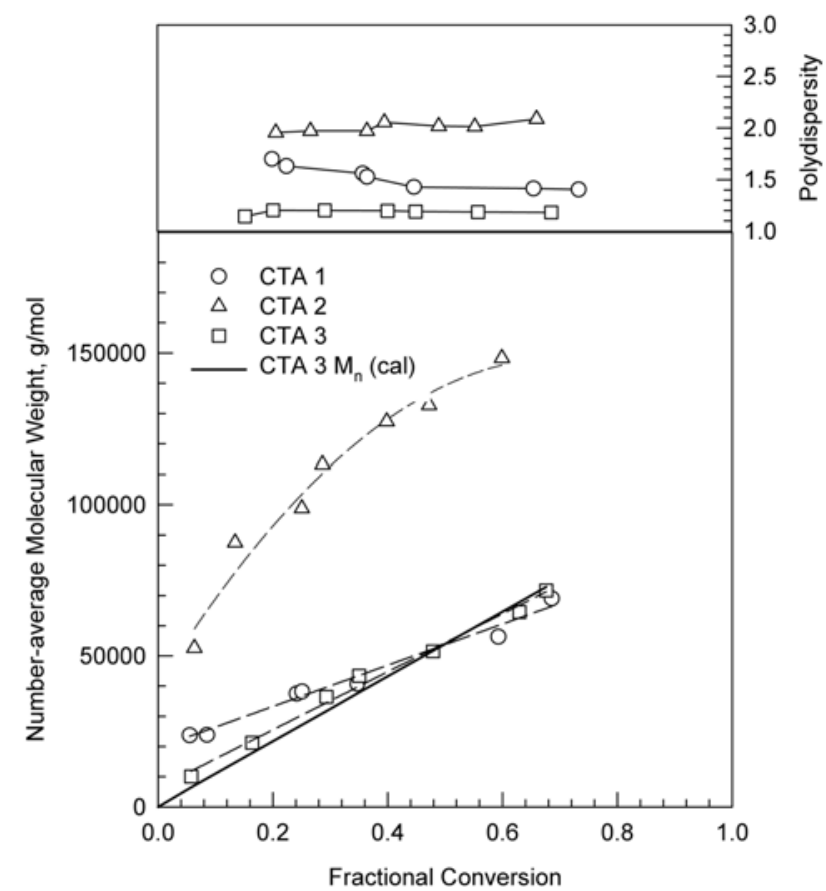

Figure 1. Polydispersity index and number-average molecular weight of polystyrene prepared by RAFT bulk polymerization with several RAFT agents. $[\mathrm{RAFT}] /[\mathrm{AIBN}]=5.0$, temperature $=$ $60{ }^{\circ} \mathrm{C}$

1.15 and 1.18 , respectively. The solid line in each figure represents the theoretically calculated molecular weights and they agree well with experimental data obtained using CTA1 and CTA3, but a substantial deviation is observed for CTA2. At the all polymerization temperatures investigated, the PDI values increase in the order of CTA2, CTA1, and CTA3.

As a result, the synthesized CTA 3 is accepted to be the best RAFT agent which successfully controls the molecular weights and the distribution. This is obviously related to the

Table I. Molecular Weight and Conversion Data for Bulk Polymerization of Styrene at 60, 70, $80{ }^{\circ} \mathrm{C}$ in the Presence of Several RAFT Agents

\begin{tabular}{ccccccc}
\hline CTA & Temp. $\left({ }^{\circ} \mathrm{C}\right)$. & Time $(\mathrm{hr})$ & Conv. & $M_{n}{ }^{a}(\mathrm{~g} / \mathrm{mol})$ & $M_{n}(\mathrm{cal}){ }^{*}(\mathrm{~g} / \mathrm{mol})$ & PDI \\
\hline \multirow{2}{*}{1} & 60 & 96 & 0.69 & 69,010 & 73,740 & 1.40 \\
& 70 & 96 & 0.67 & 6,2500 & 72,540 & 1.38 \\
& 80 & 96 & 0.73 & 69,970 & 78,550 & 1.36 \\
\hline \multirow{2}{*}{2} & 60 & 96 & 0.60 & 148,310 & 64,470 & 2.09 \\
& 70 & 96 & 0.65 & 137,870 & 70,260 & 2.33 \\
& 80 & 96 & 0.88 & 156,240 & 94,160 & 2.45 \\
\hline \multirow{2}{*}{3} & 60 & 96 & 0.68 & 71,670 & 72,780 & 1.18 \\
& 70 & 96 & 0.78 & 80,250 & 83,790 & 1.16 \\
\end{tabular}

${ }^{\circ} M_{n}(\mathrm{cal})=\mathrm{M}_{\mathrm{CTA}}+\left([\mathrm{M}]_{0} \times\right.$ Con $\left.\times \mathrm{M}_{\text {styrene }} /[\mathrm{CTA}]_{0}\right) .{ }^{a}$ Measured by GPC. 


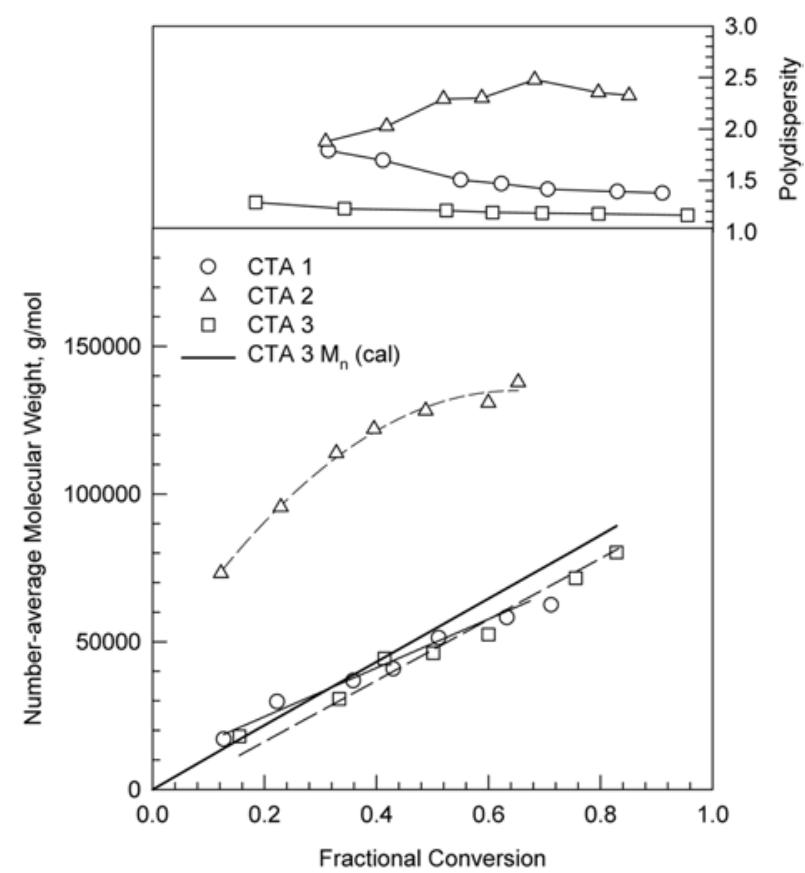

Figure 2. Polydispersity index and number-average molecular weight of polystyrene prepared by RAFT bulk polymerization at several RAFT agents. $[\mathrm{RAFT}] /[\mathrm{AIBN}]=5.0$, temperature $=70^{\circ} \mathrm{C}$.

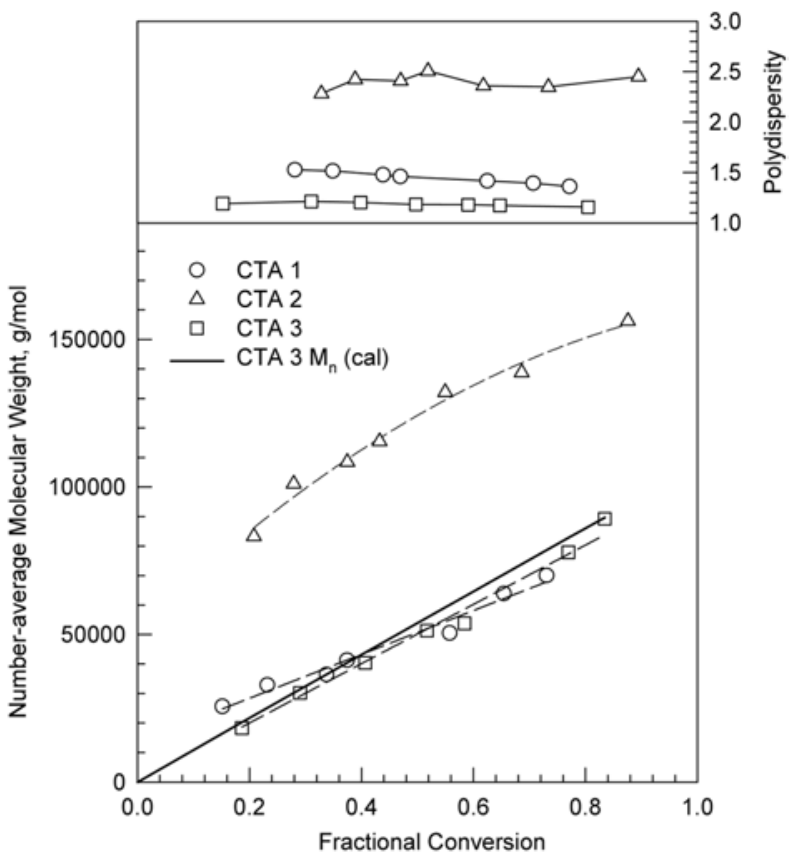

Figure 3. Polydispersity index and number-average molecular weight of polystyrene prepared by RAFT bulk polymerization with several RAFT agents at $80^{\circ} \mathrm{C}$. [RAFT] $/[\mathrm{AIBN}]=5.0$.

structure of R-group. As discussed earlier, the chemical structure of the RAFT agent, in particular, the chemical structures of the G- and Z-group strongly affects the living nature of the polymerization. Several researchers have synthesized various RAFT agents with different chemical structures and reported that the R-group having a stabilizing intermediate radical and highly steric and polar moiety is efficient in controlling the RAFT polymerization due to facile addition-fragmentation and re-initiation process. ${ }^{24,25}$

The RAFT agents used in this study have not only the electron-withdrawing group, but also the carboxyl group that causes delocalization of electrons. Although CTA1 has a carboxyl acid group, the polymerization is hardly achieved since the intermediate radical transfer is limited only to the $\alpha$-carbon, that is due to the absence of a bulky phenyl group between sulfur atom and carboxyl group. Although the molecular weight linearly increases with the conversion and the values are similar to those of the theoretically calculated ones, the PDI approaches approximately 1.4. This result implies that the polymerization is moderately controlled in the presence of CTA1.

Since the CTA2 consists of linear aliphatic carbons in Rgroup, it does not satisfy the high degree of steric factor and have deficient electron-withdrawing ability. As the conversion increases, the increase in molecular weight is not linear and the values of approximately 73,000-90,000 g/mol between 60 and $80^{\circ} \mathrm{C}$ are unusually higher than those obtained from a typical RAFT polymerization. The PDI values are ranging in $2.09 \sim 2.33$ at the above polymerization temperatures. These $M_{n}$ and PDI values are close to those of conventional free-radical polymerization rather than RAFT polymerization. As a result, CTA2 owing a linear aliphatic structure of $\mathrm{R}$-group is not sufficient to lead a living nature in this polymerization.

In the case of CTA3, the best performance is achieved because the intermediate radical on the $\alpha$-carbon can be stabilized by bulky phenyl and electron-delocalizing carboxyl acid group. Therefore, the experimentally obtained $M_{n}$ fairly matches the theoretically calculated line and the PDI is well controlled to be 1.15 . This result indicates that the CTA3 satisfying bulky and radical-stabilizing property in R-group serves as a good RAFT agent in the bulk polymerization of styrene.

Relationship between Temperature and Structure of RAFT Agent. Figures 4 and 5 show the kinetics $\left(\ln \left([\mathrm{M}]_{0} /\right.\right.$ [M]) vs reaction time) of bulk polymerization of styrene with $[\mathrm{RAFT}] /[\mathrm{AIBN}]=5.0$ at 60,70 and $80^{\circ} \mathrm{C}$ using CTA1 and CTA3, respectively. Although it is not presented for the CTA2, which poorly controls the polymerization, it is observed that the polymerization kinetics is greatly affected by the reaction temperature. For CTA1 and CTA3 as moderate and good RAFT agents, respectively, the polymerization kinetics shows a similar behavior. At $60^{\circ} \mathrm{C}$, the polymerization kinetics for CTA1 and CTA3 are much slower than those at 70 and $80^{\circ} \mathrm{C}$. However, the quite similar kinetics for CTA 1 and CTA 3 are observed at 70 and $80^{\circ} \mathrm{C}$ in Figures 4 and 5 . If the polymerization obeys a mechanism of gen- 


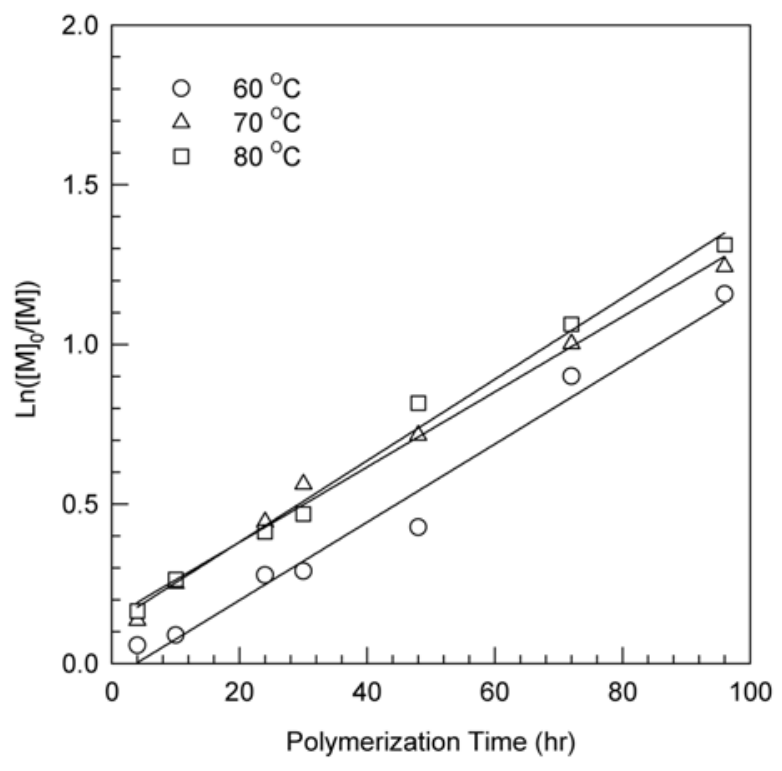

Figure 4. Polymerization kinetics of bulk polymerization of styrene using acetic acid dithiobenzoate (CTA1) at several temperatures. $[\mathrm{RAFT}] /[\mathrm{AIBN}]=5.0$.

eral free-radical polymerization, the higher temperature would significantly enhance the polymerization rate. In typical free-radical polymerizations, temperature variation

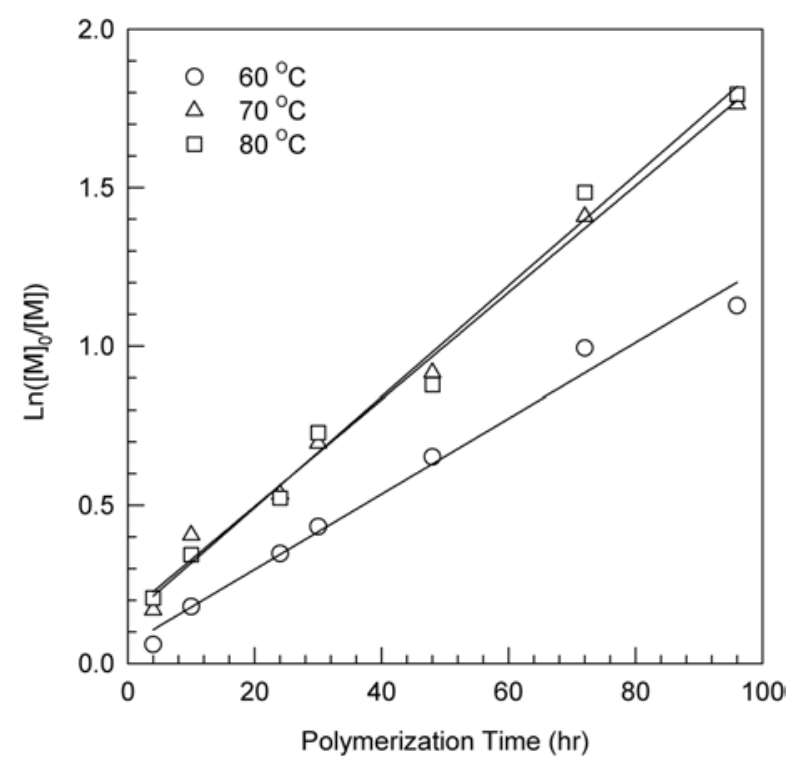

Figure 5. Polymerization kinetics of bulk polymerization of styrene using 4-toluic acid dithiobenzoate (CTA3) at several temperatures. $[\mathrm{RAFT}] /[\mathrm{AIBN}]=5.0$.

affects the rates of decomposition of initiator, termination of growing radical, and mostly propagation of monomer, which result in uncontrolled molecular weight and molecular

Table II. Effect of the Amount of RAFT Agents on the Bulk Polymerization of Styrene at $80{ }^{\circ} \mathrm{C}$

\begin{tabular}{|c|c|c|c|c|c|c|}
\hline CTA & $\begin{array}{c}{[\mathrm{CTA}] /[\mathrm{AIBN}]} \\
([\text { Styrene]/[CTA] }]\end{array}$ & Time (hr) & Con. & $M_{n}{ }^{a}(\mathrm{~g} / \mathrm{mol})$ & $M_{n}^{b}(\mathrm{~g} / \mathrm{mol})$ & PDI \\
\hline \multirow{6}{*}{1} & \multirow{3}{*}{$\begin{array}{c}2^{c} \\
(2570.7)\end{array}$} & 10 & 0.25 & 56,096 & 66,980 & 1.52 \\
\hline & & 48 & 0.59 & 84,864 & 156,711 & 1.46 \\
\hline & & 96 & 0.82 & 99,592 & 209,458 & 1.43 \\
\hline & \multirow{3}{*}{$\begin{array}{c}5^{d} \\
(1029.9)\end{array}$} & 10 & 0.23 & 32,898 & 25,071 & 1.51 \\
\hline & & 48 & 0.56 & 50,493 & 59,996 & 1.42 \\
\hline & & 96 & 0.73 & 69,972 & 78,552 & 1.36 \\
\hline \multirow{6}{*}{2} & \multirow{3}{*}{$\begin{array}{c}2 \\
(2570.7)\end{array}$} & 10 & 0.33 & 128,141 & 94,289 & 2.41 \\
\hline & & 48 & 0.64 & 170,316 & 187,167 & 2.49 \\
\hline & & 96 & 0.88 & 210,235 & 255,998 & 2.37 \\
\hline & \multirow{3}{*}{$\begin{array}{c}5 \\
(1029.9)\end{array}$} & 10 & 0.28 & 101,126 & 30,160 & 2.42 \\
\hline & & 48 & 0.55 & 132,120 & 59,156 & 2.36 \\
\hline & & 96 & 0.88 & 156,242 & 94,155 & 2.45 \\
\hline \multirow{6}{*}{3} & \multirow{3}{*}{$\begin{array}{c}2 \\
(2570.7)\end{array}$} & 10 & 0.32 & 51,089 & 87,049 & 1.34 \\
\hline & & 48 & 0.64 & 78,523 & 172,796 & 1.45 \\
\hline & & 96 & 0.88 & 101,325 & 236,341 & 1.44 \\
\hline & \multirow{3}{*}{$\begin{array}{c}5 \\
(1029.9)\end{array}$} & 10 & 0.29 & 30,254 & 31,432 & 1.21 \\
\hline & & 48 & 0.58 & 53,788 & 62,924 & 1.18 \\
\hline & & 96 & 0.83 & 89,214 & 89,678 & 1.15 \\
\hline
\end{tabular}

${ }^{a}$ Measured by GPC. ${ }^{b}$ Calculated value. ${ }^{c} 0.0272$ for the $[\mathrm{CTA}] /[\mathrm{AIBN}]=2 .{ }^{d} 0.068$ for the $[\mathrm{CTA}] /[\mathrm{AIBN}]=5$. 
weight distribution of final products. Therefore, same kinetics for CTA 1 and CTA 3 at 70 and $80{ }^{\circ} \mathrm{C}$ indicate that the polymerization is predominantly taking place by the reversible addition-fragmentation mechanism as seen in Scheme I. In our previous studies on the emulsion and dispersion polymerization using CTA3, the temperature significantly affected the polymerization characteristics. ${ }^{20,23}$

Concentration of RAFT Agents and Their Structure. Since the polymerization at $80^{\circ} \mathrm{C}$ gave a satisfactory living nature of polymerization and lowest PDI in the preceding sections, we used this temperature to study the relationship between the structure and required amount of RAFT agent to give better living characteristics. The 2 and 5 molar ratios of each RAFT agent to AIBN were used to obtain substantially high conversion for $96 \mathrm{hrs}$. The polymerization results are summarized in Table II.

In the case of CTA2, poorly controlled molecular weight and the PDI are obtained for 2 and 5 molar ratios of $[\mathrm{RAFT}] /[\mathrm{AIBN}]$ due to its linearly aliphatic R-group. In Figures 6 and 7, the $M_{n}$ and PDI as a function of the fractional conversion are demonstrated for the bulk polymerization of styrene with 2 and 5 molar ratios of [RAFT]/[AIBN] for CTA 1 and CTA 3 at $80^{\circ} \mathrm{C}$, respectively. In Figure 6 , when the molar ratio of [RAFT]/[AIBN] is 2.0, the theoretically calculated $M_{n}$ shows a significant deviation from the experimentally obtained values. However, as the molar ratio of $[\mathrm{RAFT}] /[\mathrm{AIBN}]$ raised to 5.0, the theoretically calculated $M_{n}$ values are fairly close to the experimental data. In addi-

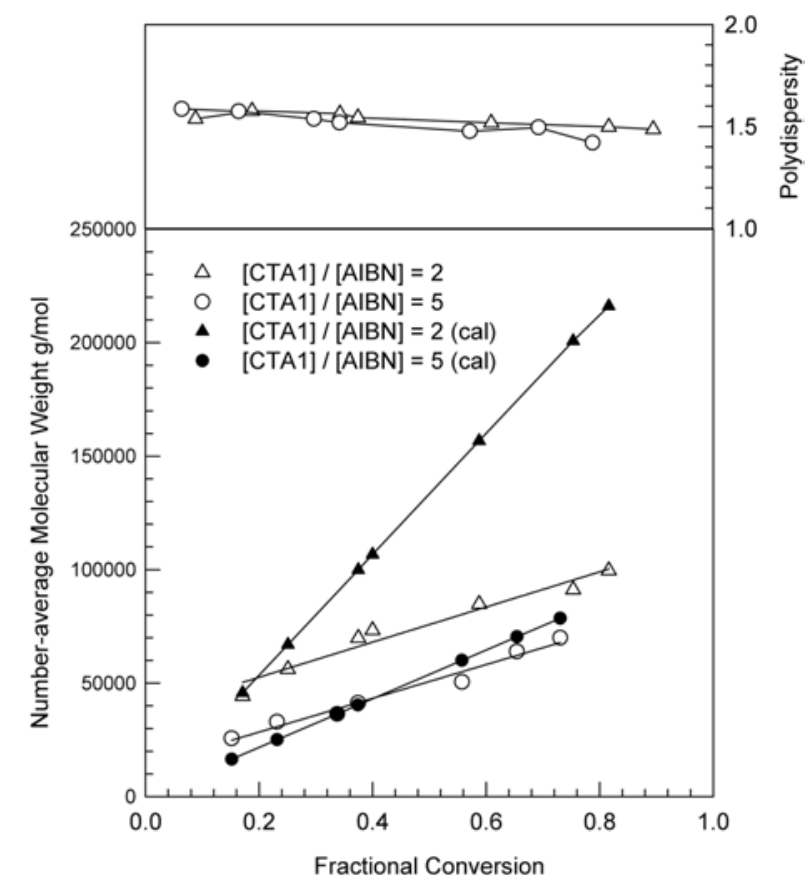

Figure 6. Polydispersity index and number-average molecular weight of polystyrene prepared by RAFT bulk polymerization using CTA 1 at $80^{\circ} \mathrm{C}$. [RAFT] $/[\mathrm{AIBN}]=2.0$ and 5.0.

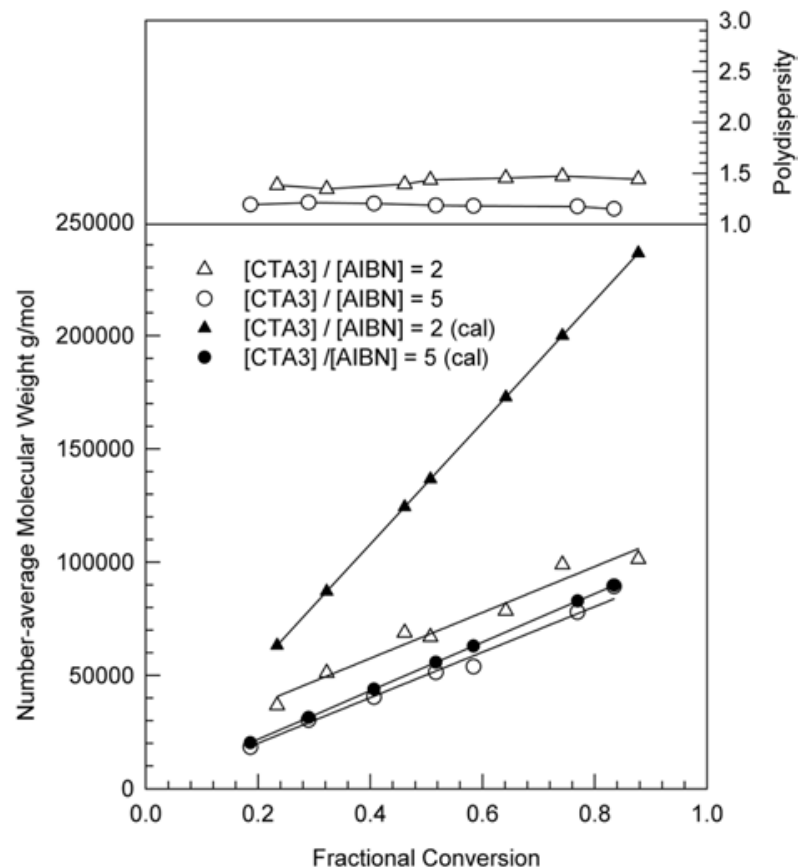

Figure 7. Polydispersity index and number-average molecular weight of polystyrene prepared by RAFT bulk polymerization using CTA3 at $80^{\circ} \mathrm{C}$. $[\mathrm{RAFT}] /[\mathrm{AIBN}]=2.0$ and 5.0.

tion, there is no significant difference (from 1.43 to 1.36 ) in the PDI between 2.0 and 5.0 of the molar ratio of [CTA1]/ [AIBN]. This implies that the increment of CTA1 higher that 5.0 molar ratio does not affect the PDI further due to poorly controlled reversible addition-fragmentation process as discussed earlier. This phenomenon is pronouncedly enhanced for CTA3 in Figure 7. The PDI is significantly reduced from 1.44 to 1.15 with increasing [CTA3]/[AIBN] from 2.0 to 5.0 due to bulky and intermediate radical stabilizing ability of CTA3. It is noted that the extrapolated molecular weights at zero polymerization time show that they pass through origin only when a RAFT agent works well. In this system, when $[\mathrm{CTA} 3] /[\mathrm{AIBN}]=5.0$, the extrapolation pass through the origin as seen in Figure 7.

Based on these investigations, it could be concluded that the R-group structure of RAFT agent carrying carboxyl polar end functionality plays an important role in RAFT bulk polymerization of styrene. As the bulky and radical stabilizing properties are enhanced, the RAFT polymerization is better controlled since reversible addition-fragmentation process is dominated by the structure of R-group. Furthermore, it is found that the R-group structure interplays with the concentration of RAFT agents and temperature of the polymerization.

\section{Conclusions}

Three dithioester-derived carboxyl acid functionalized 
RAFT agents were used in bulk RAFT polymerization of styrene in order to study the effects of R-group structure on the living nature of the polymerization. The agents used were acetic acid dithiobenzoate (CTA1), butanoic acid dithiobenzoate (CTA2), and 4-toluic acid dithiobenzoate (CTA3). CTA2, which has linear aliphatic R-group, is found to poorly control the polymerization, resulting in non-linear molecular weight evolution and high PDI regardless of its concentration at all the temperatures. CTA1 having a single $\alpha$-carbon moderately controls the polymerization resulting in a satisfactory molecular weight and intermediate PDI with increasing concentration. For CTA3, the best living nature of the polymerization is achieved with fairly excellent molecular weight evolution and low PDI with increasing concentration. Furthermore, it is found that CTA1 and CTA3 are being well activated above $70^{\circ} \mathrm{C}$, indicating a particular temperature is necessary for an efficient reversible addition-fragmentation process. Therefore, it could be concluded that the R-group structure of RAFT agent carrying carboxyl polar end functionality plays in important role in RAFT polymerization. As the bulky and radical stabilizing properties are enhanced, the RAFT polymerization is better controlled since reversible addition-fragmentation process is dominated by the structure of R-group. In addition, it is found that the R-group structure interplays with the concentration of RAFT agents and temperature of the polymerization.

Acknowledgements. This work is financially supported by the Korea Research Foundation (KRF-2003-42-D00056) in 2003-2005.

\section{References}

(1) T. Otsu and M. Yoshida, Macromol. Rapid Commun., 3, 127 (1982).

(2) K. Matyjaszewski and T. P. Davis, Eds., Handbook of Radical Polymerization, Wiley, Hoboken, 2002.

(3) M. K. Georges, R. P. N. Veregin, P. M. Kazmaier, and G. K. Hamer, Macromolecules, 26, 2987 (1993).

(4) D. H. Solomon, E. Rizzardo, and P. Cacioli, US 4581429 (1986).

(5) J. S. Wang, and K. Matyjaszewski, J. Am. Chem. Soc., 117, 5614 (1995).

(6) K. Matyjaszewski and J. S. Wang, Macromolecules, 8, 7901 (1995).

(7) M. Kato, M. Kamigato, M. Sawamoto, and T. Higashimura,
Macromolecules, 28, 1721 (1995).

(8) W. Xu, X. Zhu, Z. Cheng, and J, Lu, Macromol. Res., 12, 32 (2004).

(9) J. Chiefari, Y. K. Chong, F. Ercole, J. Krstina, J. Jeffery, T. P. T. Le, R. T. A. Mayadunne, G. F. Meijs, C. L. Moad, G. Moad, E. Rizzardo, and S. H. Thang, Macromolecules, 31, 5559 (1998).

(10) D. G. Hawthorne, G. Moad, E. Rizzardo, and S. H. Thang, Macromolecules, 32, 5457 (1999).

(11) A.Goto, K. Sato,Y. Tsujii, T. Fukuda, G. Moad, E. Rizzardo, and S. H. Thang, Macromolecules, 34, 402 (2001).

(12) B. Y. K. Chong, T. P. T. Le, G. Moad, E. Rizzardo, and S. H. Thang, Macromolecules, 32, 2071 (1999).

(13) R. T. A. Mayadunne, E. Rizzardo, J. Chiefari, J. Krstina, G. Moad, A. Postma, and S. H. Thang, Macromolecules, 33, 243 (2000). S. Perrier, C. Barner-Kowollik, J. F Quinn, P. Vana, T. P. Davis, Macromolecules, 35, 8300 (2002).

(14) C. Barner-Kowollik, J. F. Quinn, T. L. U. Nguyen, J. P. A. Heuts, and T. P. Davis, Macromolecules, 34, 7849 (2001).

(15) M. Destarac, D. Charmot, X. Franck, and S. Z. Zard, Macromol. Rapid Commun., 21, 1036 (2000).

(16) R. Severac, P. Lacroix-Desmazes, and B. Boutevin, Polym. Int., 51, 1117 (2002).

(17) G. Moad, J. Chiefari, Y. K. Chong, J. Krstina, R. T. A. Mayadunne, A. Postma, E. Rizzardo, and S. H. Thang, Polym. Int., 49, 993 (2000).

(18) C. Barner-Kowollik, J. F. Quinn, D. R. Morseley, and T. P. Davis, J. Polym. Sci.; Part A Chem., 39, 1353 (2001).

(19) M. S. Donovan, A. B. Lowe, B. S. Sumerlin, C. L. McCormik, Macromolecules, 34, 6561 (2001). M. S. Donovan, A. B. Lowe, B. S. Sumerlin, and C. L. McCormick, Macromolecules, 35, 8300 (2002).

(20) M. S. Donovan, T. A. Sanford, A. B. Lowe, B. S. Sumerlin, Y. Mitsukami, and C. L. McCormik, Macromolecules, 35, 4570 (2002).

(21) S. E. Shim, H. Lee, and S. Choe, Macromolecules, 37, 5565 (2004).

(22) S. R. Ramadas and P. S. Srinivasan, J. Prakt. Chemie, 319, 169 (1977).

(23) S. E. Shim, Y. Shin, H. Lee, and S. Choe, Polym. Bull., 51, 209 (2003).

(24) S. E. Shim, H. Jung, H. Lee, J. Biswas, and S. Choe, Polymer, 44, 5563 (2003).

(25) Y. K. Chong, J. Krstina, T. P. T. Le, G. Moad, A. Postma, E. Rizzardo, and S. H. Thang, Macromolecules, 36, 2256 (2003).

(26) J. Chiefari, R. T. A. Mayadunne, C. L. Moad, G. Moad, E. Rizzardo, A. Postma, M. A. Skidmore, and S. H. Thang, Macromolecules, 36, 2273 (2003). 